\title{
Chemical Functionalization, Self-Assembly, and Applications of Nanomaterials and Nanocomposites
}

\author{
Tifeng Jiao, ${ }^{1}$ Xingbin Yan, ${ }^{2}$ Lavinia Balan, ${ }^{3}$ Andrey L. Stepanov, ${ }^{4}$ \\ Xinqing Chen, ${ }^{5}$ and Michael $\mathrm{Z}$. $\mathrm{Hu}^{6}$ \\ ${ }^{1}$ School of Environmental and Chemical Engineering, Yanshan University, Qinhuangdao 066004, China \\ ${ }^{2}$ Laboratory of Clean Energy Chemistry and Materials, Lanzhou Institute of Chemical Physics, Chinese Academy of Sciences, \\ Lanzhou 730000, China \\ ${ }^{3}$ Institute of Materials Science of Mulhouse (IS2M), rue Jean Starcky BP 2488, \\ 68057 Mulhouse cedex, France \\ ${ }^{4}$ Kazan Physical-Technical Institute, Russian Academy of Sciences, Kazan 420029, Russia \\ ${ }^{5}$ Department of Chemical and Biomolecular Engineering, Hong Kong University of Science and Technology, \\ Clear Water Bay, Kowloon, Hong Kong \\ ${ }^{6}$ Energy and Transportation Science Division, Oak Ridge National Laboratory, Oak Ridge, TN 37831, USA
}

Correspondence should be addressed to Xingbin Yan; xbyan@licp.cas.cn

Received 16 January 2014; Accepted 16 January 2014; Published 12 May 2014

Copyright (C) 2014 Tifeng Jiao et al. This is an open access article distributed under the Creative Commons Attribution License, which permits unrestricted use, distribution, and reproduction in any medium, provided the original work is properly cited.

Nanomaterials and nanocomposites have attracted considerable interest for application in various areas due to their unique properties. The functionalization and bottom-up assemblies of nanomaterials and subsequent applications of well-defined micro/nanostructures have multiple important impacts upon nanoscience and nanotechnology. The sophisticated nanostructures with multiple components have deepened the insight of nanomaterials and enriched the content of supramolecular chemistry. It can be predicted that, due to the growth of understanding of the rules in the nanoscale, our dream to manipulate molecules to achieve the precisely tailored organic/inorganic nanostructures could be realized in future.

This special issue addresses the research studies on chemical functionalization, self-assembly, and applications of nanomaterials and nanocomposites. It contains twentyfour articles including two reviews and twenty-two research articles. It is used to create new functional nanomaterials and nanocomposites with a variety of sizes and morphologies such as $\mathrm{Zn} / \mathrm{Al}$ layered double hydroxide, tin oxide nanowires, $\mathrm{FeOOH}$-modified anion resin, $\mathrm{Au}$ nanoclusters silica composite nanospheres, Ti-doped $\mathrm{ZnO}$ sol-composite films, $\mathrm{TiO}_{2} / \mathrm{ZnO}$ composite, graphene oxide nanocomposites, $\mathrm{LiFePO}_{4} / \mathrm{C}$ nanocomposites, and chitosan nanoparticles. These nanomaterials and nanocomposites have widespread applications in tissue engineering, antitumor, sensors, photoluminescence, electrochemical, and catalytic properties. In addition, this themed issue includes some research articles about self-assembly systems covering organogels and Langmuir films. Furthermore, B. Blasiak et al. performed a literature survey on the recent advances in production, functionalization, toxicity reduction, and application of nanoparticles in cancer diagnosis, treatment, and treatment monitoring. P. Colson et al. performed a literature survey on the recent advances in nanosphere lithography due to its compatibility with wafer-scale processes as well as its potential to manufacture a wide variety of homogeneous one-, two-, or three-dimensional nanostructures.

\section{Acknowledgments}

The guest editors would like to express sincere appreciation to all the authors for their contribution. Moreover, thanks are 
extended to all reviewers for their time and for enhancing the quality of these papers.

Tifeng Jiao

Xingbin Yan

Lavinia Balan

Andrey L. Stepanov

Xinqing Chen

Michael Z. Hu 

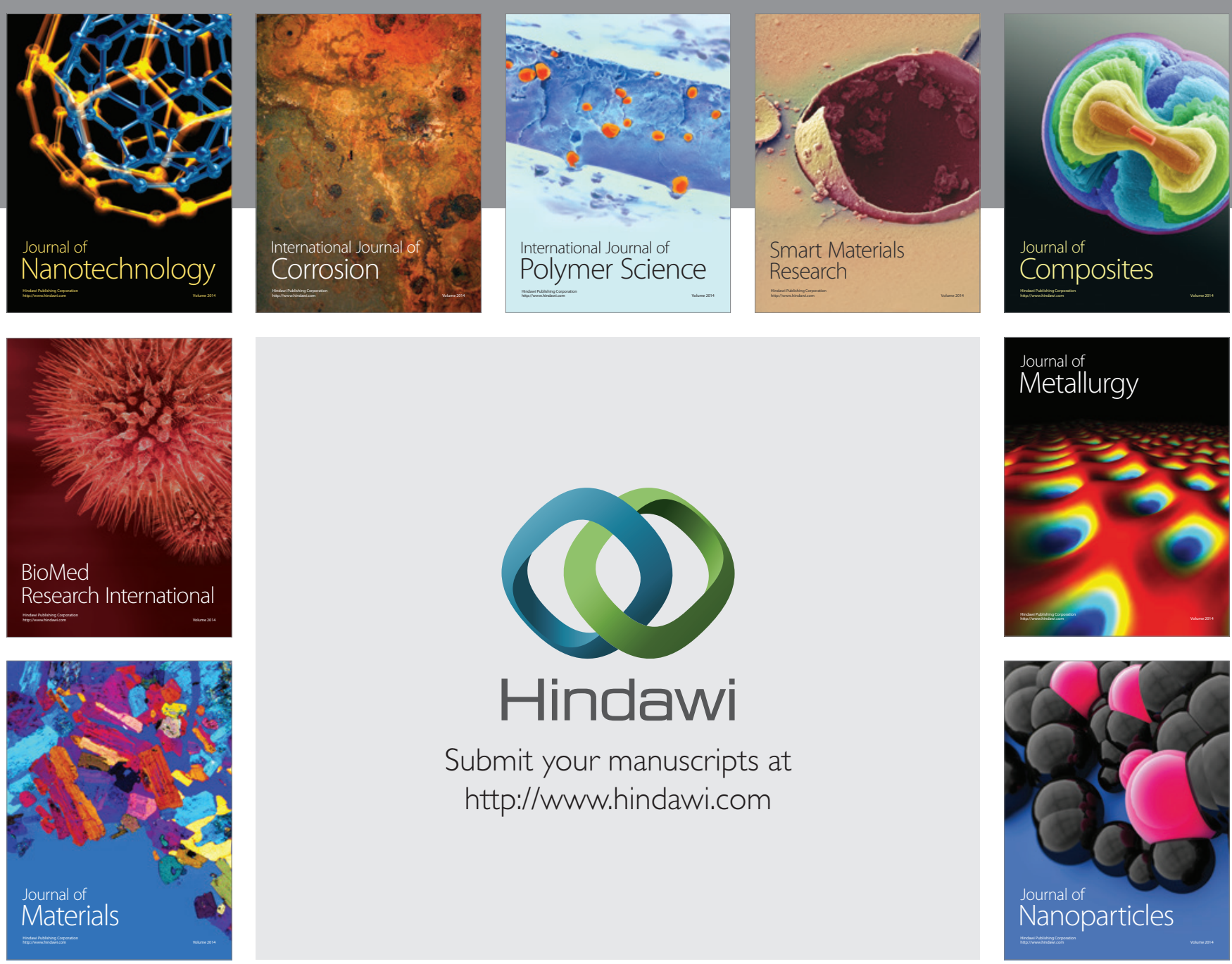

Submit your manuscripts at http://www.hindawi.com
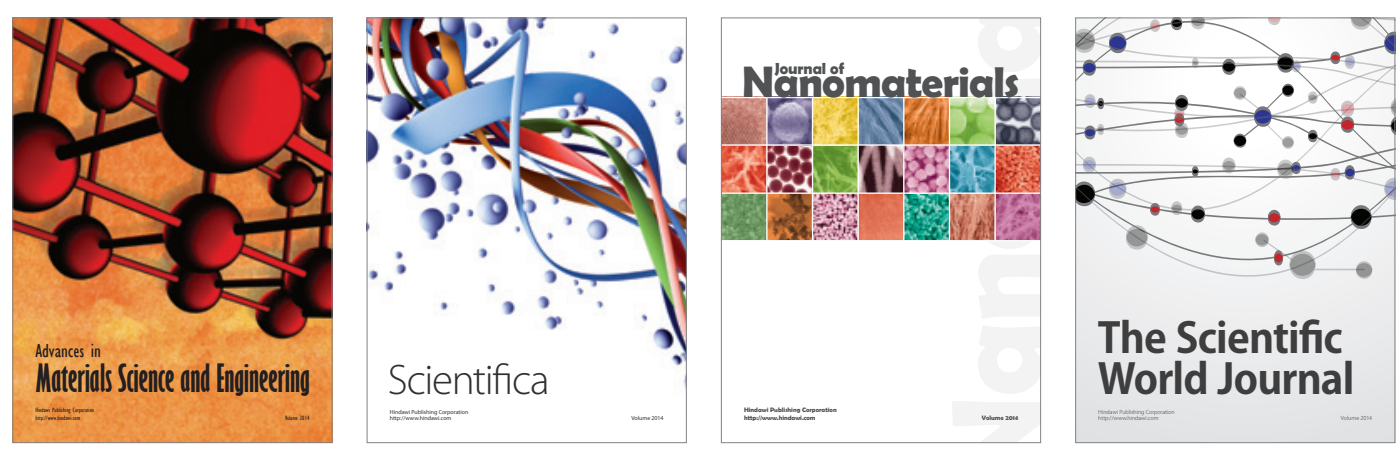

\section{The Scientific World Journal}
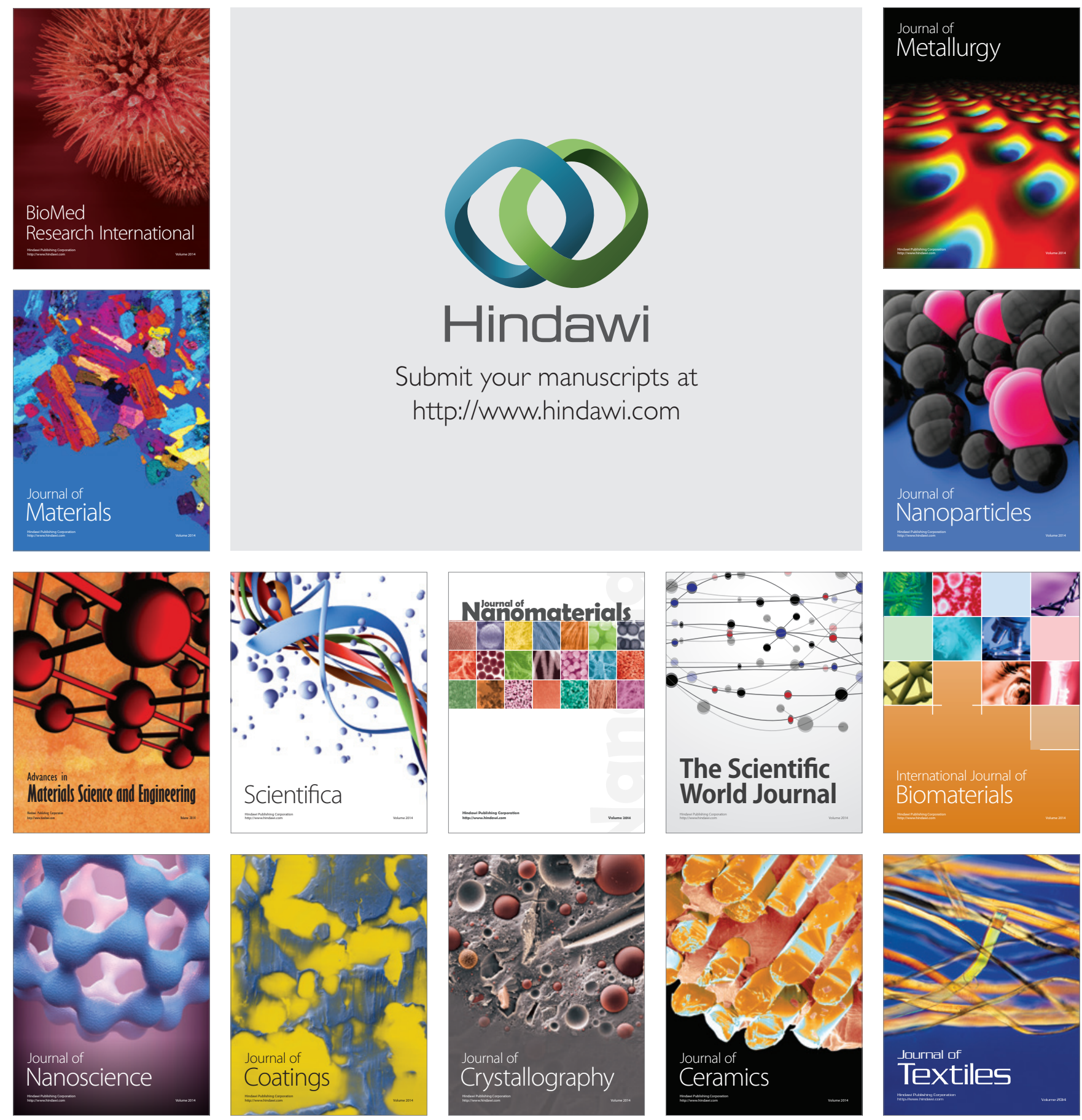